\title{
Utility and safety of sole electromagnetic navigation bronchoscopy under moderate sedation for lung cancer diagnosis
}

\author{
Yeon Wook Kim ${ }^{1,2}$, Hyung-Jun Kim ${ }^{1,2}$, Myung Jin Song ${ }^{1,2}$, Byoung Soo Kwon ${ }^{1,2}$, Sung Yoon Lim ${ }^{1,2}$, \\ Yeon Joo Lee ${ }^{1,2}$, Jong Sun Park ${ }^{1,2}$, Young-Jae Cho ${ }^{1,2}$, Ho Il Yoon ${ }^{1,2}$, Jae Ho Lee ${ }^{1,2}$, Choon-Taek Lee ${ }^{1,2}$ \\ ${ }^{1}$ Department of Internal Medicine, Seoul National University College of Medicine, Seoul, Korea; ${ }^{2}$ Division of Pulmonary and Critical Care \\ Medicine, Department of Internal Medicine, Seoul National University Bundang Hospital, Seongnam, Republic of Korea \\ Contributions: (I) Conception and design: All authors; (II) Administrative support: YW Kim, HI Yoon, CT Lee; (III) Provision of study materials or \\ patients: YW Kim, HI Yoon; (IV) Collection and assembly of data: All authors; (V) Data analysis and interpretation: All authors; (VI) Manuscript \\ writing: All authors; (VII) Final approval of manuscript: All authors. \\ Correspondence to: Yeon Wook Kim. Division of Pulmonary and Critical Care Medicine, Department of Internal Medicine, Seoul National University \\ Bundang Hospital, 173-82 Gumi-Ro, Bundang-gu, Seongnam 13620, Republic of Korea. Email: kimyw@snu.ac.kr.
}

Background: Electromagnetic navigation bronchoscopy (ENB) is an emerging technique for diagnosing pulmonary lesions. However, limited data is available on its sole utility under a least invasive setting without general anesthesia. We aimed to evaluate the diagnostic performance and safety of sole ENB under moderate sedation for diagnosing pulmonary lesions suspicious for lung cancer and to determine clinical factors associated with a better diagnostic yield.

Methods: We performed a retrospective analysis of consecutive patients who underwent sole ENB under moderate sedation for lung lesion biopsy between August 2016 and June 2021 at Seoul National University Bundang Hospital, a tertiary center in South Korea. Diagnostic yield of the ENB-guided biopsy, safety endpoints defined by the incidence and severity of associated complications, and factors associated with higher diagnostic yield were evaluated.

Results: A total of 94 patients were evaluated. The final diagnostic yield of ENB was $81.5 \%(75 / 92)$, excluding two indeterminate cases. The diagnostic yield ranged from $79.8 \%$ to $81.9 \%$ assuming all indeterminate cases were false-negatives $(79.8 \%)$ and true-negatives $(81.9 \%)$. The sensitivity and specificity for malignancy were $77.6 \%$ (ranging from $75.6 \%$ to $77.6 \%$ ) and $100 \%$, respectively. Any-grade pneumothorax occurred in $4.3 \%$ of the patients, and $2.1 \%$ developed pneumothorax requiring additional intervention. Multivariable analyses identified the presence of a class 2 bronchus sign as the only significant predictor for a higher diagnostic yield (odds ratio $=4.83,95 \% \mathrm{CI}$ : 1.16-20.12). The diagnostic yield of ENB among those with class 2 bronchus sign was $89.8 \%(53 / 59)$.

Conclusions: Sole ENB under moderate sedation for diagnosing pulmonary lesions displayed a good diagnostic yield and safety profile, thus confirming its utility in a least-invasive setting. Moreover, sole ENB could be possibly be superior to transthoracic needle aspiration for diagnosing lesions with class 2 bronchus sign accounting for similar yields and lower complication rates.

Keywords: Electromagnetic navigation bronchoscopy (ENB); lung cancer; minimally invasive; diagnostic yield; moderate sedation

Submitted Oct 18, 2021. Accepted for publication Jan 06, 2022.

doi: $10.21037 /$ tlcr-21-846

View this article at: https://dx.doi.org/10.21037/tlcr-21-846

^ ORCID: 0000-0001-8333-4812. 


\section{Introduction}

Lung cancer is the leading cause of cancer-related deaths worldwide (1). Widespread efforts have focused on developing effective screening methods to detect and treat lung cancer at an early stage, and the increased use of lowdose computed tomography (LDCT) for lung cancer screening has led to a considerable increase in the detection of lung lesions that require diagnostic evaluation (2). According to clinical practice guidelines, the least invasive approach with the highest yield for biopsy is recommended for patients with pulmonary lesions suspicious of lung cancer $(3,4)$. For peripheral lesions, transthoracic needle aspiration (TTNA) has been widely adopted. However, despite a good sensitivity of approximately $90 \%$, TTNA accounts for a relatively high risk of pneumothorax among $>18 \%$ of the biopsied cases $(5,6)$. Recently, advances in bronchoscopy techniques to improve the diagnostic yield and safety have made bronchoscopic biopsy more appealing $(7,8)$. Electromagnetic navigation bronchoscopy (ENB) allows physicians to access peripheral lung lesions through a minimally invasive method by using an image-guided flexible catheter and a dedicated navigation software system. ENB is recommended for lesions that are difficult to reach with conventional bronchoscopy alone $(3,9)$.

Researchers have conducted several studies to establish the diagnostic yield and safety as well as to facilitate patient selection for ENB. Despite most studies being retrospective and single centered, one large multicenter prospective study (10), followed by a recent meta-analysis, demonstrated that ENB-guided diagnosis provides good accuracy for diagnosing malignancy with a yield of approximately $75 \%$ and a procedural complication rate lower than $3 \%$ for pneumothorax (11). However, most of the previous studies involved patients who underwent ENB under general anesthesia, and a substantial proportion underwent multiple procedures for guidance other than ENB such as radial endobronchial ultrasound (r-EBUS) and/or fluoroscopy (10,12-14). Data on the performance of the sole ENB without general anesthesia are limited. General anesthesia for bronchoscopic procedures is reported to be associated with a greater need for the post-procedure escalation of care (15). Therefore, the sole use of ENB under moderate sedation that yields promising diagnostic performance and safety would make the procedure minimally invasive and feasible even in clinical settings with limited access to general anesthesia. Moreover, predictors for high diagnostic yield under this minimally invasive setting for ENB must be determined. Therefore, we aimed to evaluate the diagnostic performance and safety of sole ENB under moderate sedation for diagnosing pulmonary lesions suspicious for lung cancer, and to determine clinical factors associated with better diagnostic yield. We present the following article in accordance with the STROBE reporting checklist (available at https://tlcr.amegroups.com/article/view/10.21037/tlcr$21-846 / \mathrm{rc})$.

\section{Methods}

\section{Study design and participants}

We designed a single-center, retrospective cohort study of adults $\geq 18$ years of age who underwent ENB for lung lesion biopsy between August 2016 and June 2021 at Seoul National University Bundang Hospital, a tertiary center in South Korea. Consecutive subjects presenting with a lung lesion that required pathologic evaluation under suspicion for malignancy and candidates for an elective ENB procedure, based on the discretion of the attending specialist in the pulmonary division were enrolled. Patients who received ENB under general anesthesia and those who underwent concurrent r-EBUS or fluoroscopy were excluded. Moreover, we excluded those who were initially planned to undergo ENB but successfully underwent biopsy from a visible endobronchial tumor lesion that precluded the need for electromagnetic navigation. This study was conducted in accordance with the declaration of Helsinki (as revised in 2013), and was approved by the Institutional Review Board of Seoul National University Bundang Hospital (IRB No. B-2106-693-104). The Institutional Review Board waived the need for written informed consent from participants, considering the retrospective nature of this study.

\section{ENB procedures}

On the ENB procedure day, all patients planned for ENB underwent inspiration/expiration chest computed tomography $(\mathrm{CT})$ to index with navigation platforms and reconstruct virtual airway routes prior to the procedure. At Seoul National University Bundang Hospital, moderate sedation is the routine method of anesthesia for diagnostic bronchoscopic techniques. Besides studies performed under general anesthesia owing to unavoidable medical conditions or intraoperative studies, all ENB procedures were performed under moderate sedation by administration 
of $2-3 \mathrm{mg}$ of midazolam and $25-50 \mu \mathrm{g}$ of fentanyl intravenously at the procedure onset. The performing physician may have administered an additional dose of midazolam or fentanyl during the procedure for adequate sedation. The routine procedure of ENB involved the use sole ENB without additional complementary tools, such as r-EBUS and fluoroscopy, unless the performing physician decided to use additional supportive techniques.

All ENB procedures were performed by one of the nine pulmonologists (HIY, YJC, JSP, YJL, SYL, BSK, YWK, MJS, and HJK, each of whom had at least 3 years of experience with bronchoscopic procedures), using the Spin Thoracic Navigation System (SYS-4230 K; Veran Medical, St. Louis, MO, USA) with a bronchoscope having an outer diameter of $5.9 \mathrm{~mm}$ (BF-1TQ290) or $4.9 \mathrm{~mm}$ (BF-260) (both Olympus Corporation, Tokyo, Japan). The performing physician may have used one of the following tools for ENB-guided biopsy: aspirating needle or biopsy forceps. There were no other specified restrictions on additional procedural techniques (e.g., additional cytology brushing, bronchoalveolar lavage, or bronchial washing) and were subjected to the performing physician's discretion. Rapid on-site examination (ROSE) was not performed in any of the cases. Mediastinal staging of lung cancer with linear EBUS could be performed before or after the ENB procedure at the physician's discretion.

\section{Outcome measures}

Medical records documenting clinical data including demographic characteristics, radiologic findings including the size in longest diameter, solidity, distance from pleura of the target lesion in the inspiration/expiration chest CT, ENB procedural reports, pathologic reports and the final diagnoses of the biopsied lesion, and any procedureassociated complications were obtained. In cases with multiple lung lesions that underwent biopsy, the dominant lesion intended for ENB-guided biopsy was selected for the analyses. The presence of bronchus sign on CT was determined and classified according to the classification first provided by Tokoro and colleagues as follows; class 0 : the absence of a bronchus sign, class 1: an airway sits immediately adjacent to the lesion, and class 2: an airway directly aligned with the lesion $(16,17)$. Pathology results of ENB-guided samples that revealed non-malignant or indeterminate conditions were initially referred to as negative. For such cases, the attending physician made decisions regarding the follow-up and attempting other invasive procedures for pathologic evaluations. The following instances were defined as false negatives: (I) a repeat biopsy (e.g., surgical, TTNA, and bronchoscopic) that proved malignancy, (II) lesion growth was observed on follow-up CT imaging within 6 months, (III) treated as lung cancer without pathological confirmation, and (IV) lung cancer diagnosis from any other sites within 3 months.

In contrast, the following cases were defined as true negatives: (I) subsequent diagnostic tests confirmed a nonmalignant diagnosis, (II) the resolution of lesion without lung cancer treatment, and (III) no progression on CT follow-up up to 6 months. ENB-related complications were determined and classified according to the Common Terminology Criteria for Adverse Events (CTCAE) scale, ver. $5.0(18,19)$. Pneumothorax was confirmed by chest $\mathrm{X}$-rays taken post-procedurally and the day after the procedure. Severe bronchopulmonary hemorrhage was designated when a blood transfusion or intervention was indicated (CTCAE grade 2). The main outcomes of this study were the diagnostic yield of the ENB-guided biopsy and safety endpoints defined by incidence and severity of associated complications. Moreover, factors associated with higher diagnostic accuracy were evaluated.

\section{Statistical analysis}

Characteristics of participants are presented as frequencies (\%) for categorical variables and as means and standard deviations for continuous variables. The diagnostic yield of ENB was calculated for each participant as the sum of the rate of true-positive cases (for malignancy) and that of true negative cases (for malignancy) of all participants who underwent ENB-guided biopsy (10). Cases that had with initially negative results with insufficient information or follow-up duration to conclude true- or false- negative were included in a sensitivity analysis, assuming all as truenegative or false-negative cases. This provided low and high estimates of the diagnostic yield, sensitivity, specificity, positive predictive value (PPV), and negative predictive value (NPV) of ENB for diagnosing malignancy. Univariate and multivariate analyses were performed using logistic regression models to determine the factors associated with the diagnostic yield. The multivariate models were adjusted for age, sex, smoking status, and variables with $P$ values less than 0.2 in univariable analyses with exclusion of variables that showed multicollinearity. No covariates included in the regression models had missing values. The odds ratios (ORs) and 95\% confidence intervals (CIs) were calculated, 


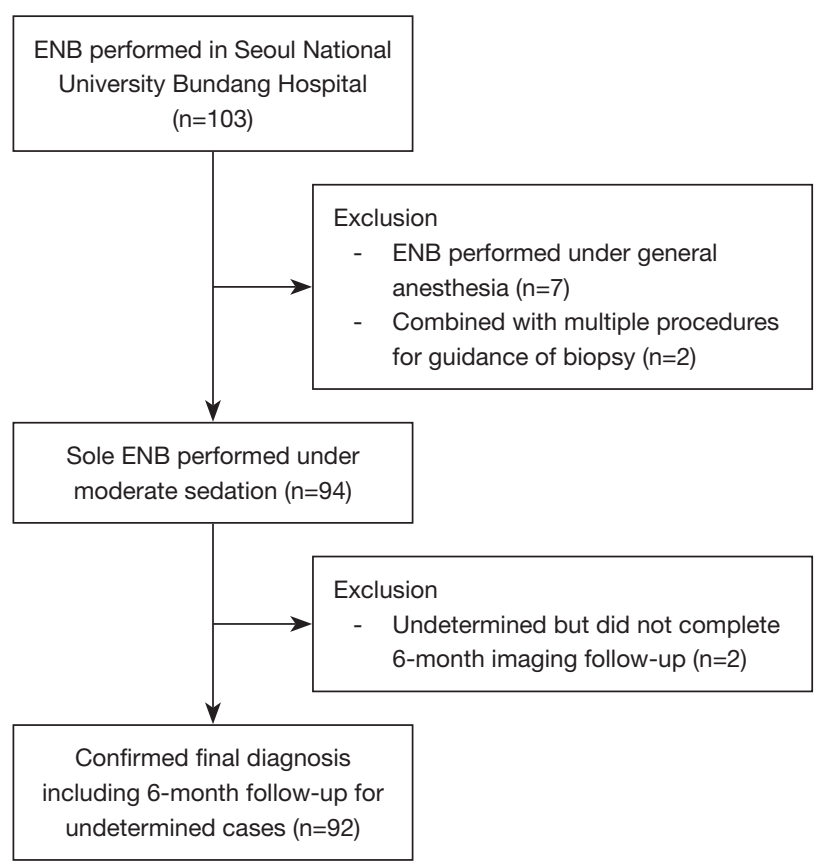

Figure 1 Flow diagram of the study population. ENB, electromagnetic navigation bronchoscopy.

and $\mathrm{P}$ values $<0.05$ were considered statistically significant. All analyses were performed using IBM SPSS Statistics 25.0 (IBM Corp., Armonk, NY, USA) and STATA, version 16.0 (StataCorp., College Station, TX, USA).

\section{Results}

\section{Participant and procedural characteristics}

Figure 1 depicts the flowchart of the study participants. During the study period, 103 patients underwent ENB. Following the exclusion of those who underwent ENB under general anesthesia or received other guidance for biopsy besides ENB, 94 patients were initially analyzed. For analyzing the final diagnostic yield, total of 92 patients were evaluated after excluding patients with initially undetermined biopsy results from ENB and without 6 months of imaging follow-up.

Table 1 summarizes the baseline characteristics of 94 patients who underwent sole ENB under moderate sedation. The average age was $68.2 \pm 9.8$ years. Of all participants, $67.0 \%$ were men, and $43.6 \%$ were neversmokers. The mean size of the targeted lesions was $34.3 \pm 14.9 \mathrm{~mm}$, with a mean distance of $23.2 \pm 15.6 \mathrm{~mm}$ from the pleura. Regarding radiologic findings, $77.7 \%$ of lesions were located in the peripheral two-thirds of the lung, and $63.8 \%$ revealed a class 2 bronchus sign. For ENB-guided biopsy, forceps biopsy was more frequently used (70.2\%) than needle aspiration biopsy $(29.8 \%)$. For moderate sedation, a mean dose of $5.2 \pm 1.5 \mathrm{mg}$ of midazolam and $71.5 \pm 28.3 \mu \mathrm{g}$ of fentanyl was administered, respectively. No other drugs were necessary to achieve adequate sedation during the procedure. There were no reports of acute toxicity related to sedative drugs.

\section{Diagnostic outcomes and safety profiles}

Figure 2 depicts the final diagnostic results of those who underwent sole ENB. ENB-guided biopsy led to a diagnosis of malignancy in 59 of the $94(62.8 \%)$ cases that underwent the procedure, but it was negative (for malignancy) in $35(37.2 \%)$ cases. Cancers diagnosed by ENB included 53 non-small cell lung cancers and two each of small cell lung cancers, metastatic carcinomas, and lymphomas. Of the 35 initially negative cases, 16 (45.7\%) and 17 (48.6\%) were eventually confirmed as true-negatives and falsenegatives, respectively. Two indeterminate cases were initially considered negative by ENB-guided biopsy and did not complete 6 months follow-up CT imaging for a nonprogressive lesion. Of the $94 \mathrm{ENB}$-guided biopsy cases, $21(22.3 \%)$ were referred for molecular testing, and all 21 biopsy specimens were adequate for additional genetic studies.

The final diagnostic yield of ENB was $81.5 \%$, calculated as the sum of true positives (for malignancy) and true negatives (for malignancy) divided by total number of ENB cases, excluding the indeterminate cases (denominator $=92$ ) (Table 2). The diagnostic yield ranged from $79.8 \%$ to $81.9 \%$ assuming all indeterminate cases were false-negatives $(79.8 \%)$ and true-negatives (81.9\%). Values of $77.6 \%$ (ranging from $75.6 \%$ to $77.6 \%$ ) and $100 \%$ were obtained as the sensitivity and specificity for malignancy, respectively.

For adverse events, any-grade pneumothorax occurred in four $(4.3 \%)$ patients who underwent ENB. The incidence of pneumothorax requiring intervention such as thoracic tube insertion, was $2.1 \%$ (2/94). Of those which pneumothorax occurred, the mean lesion size and distance from pleura was $28.3 \pm 11.9$ and $39.3 \pm 7.2 \mathrm{~mm}$, respectively. Location of the lesions was two at left upper lobe, one each at right upper lobe and right lower lobe, respectively. Three cases were diagnosed with malignancy. Bronchopulmonary hemorrhage of CTCAE grade 2 or greater occurred in $1.1 \%$ (1/94) of the patients. There were no reports of respiratory 
Table 1 Demographic, clinical, procedural characteristics of study participants

\begin{tabular}{lc}
\hline Characteristics & Total $(\mathrm{n}=94)$ \\
\hline Age, mean \pm SD & $68.2 \pm 9.8$ \\
Sex, $\mathrm{n}(\%)$ & $63(67.0)$ \\
Male & $31(33.0)$ \\
Female & \\
Smoking status at procedure, $\mathrm{n}(\%)$ & $41(43.6)$ \\
Never-smoker & $31(33.0)$ \\
Former smoker & $22(23.4)$ \\
Current smoker & $19.8 \pm 24.3$ \\
Pack years smoked, mean $\pm \mathrm{SD}$ & $34.3 \pm 14.9$ \\
Lesion size, mm, mean $\pm \mathrm{SD}$ & \\
Location of lesion, $\mathrm{n}(\%)$ & $33(35.1)$ \\
Right upper lobe & $5(5.3)$ \\
Right middle lobe & $15(16.0)$ \\
Right lower lobe & $18(19.1)$ \\
Left upper lobe & $23(24.5)$ \\
Left lower lobe & $73(77.7)$ \\
Lesion in peripheral two-thirds of the lung, $\mathrm{n}(\%)$ & $23.2 \pm 15.6$ \\
Distance from lesion to pleura, mm, mean $\pm \mathrm{SD}$ & \\
Bronchus sign on CT, $\mathrm{n}(\%)$ & \\
Class 0 & \\
Class 1 & \\
Class 2 & \\
\hline
\end{tabular}

Type of lesion, n (\%)

Solid

$87(92.6)$

Subsolid

Tool used, n (\%)

Forceps biopsy

$66(70.2)$

Needle aspiration biopsy

Sequential EBUS-TBNA for mediastinal staging, $\mathrm{n}(\%)$

Total dose of sedatives, mean $\pm \mathrm{SD}$

Midazolam, mg

$5.2 \pm 1.5$

Fentanyl, $\mu \mathrm{g}$

$71.5 \pm 28.3$

Total procedure time, min, mean $\pm S D$

SD, standard deviation; CT, computed tomography; EBUS, endobronchial ultrasound; TBNA, transbronchial needle aspiration. failure events or procedure-related deaths.

\section{Predictors of higher diagnostic yield}

Univariable and multivariable analyses of factors associated with higher diagnostic yield of ENB are described in Table 3. In the univariable analyses, larger lesion size $(\mathrm{OR}=1.05$, 95\% CI: $1.00-1.10)$ and the presence of a class 2 bronchus sign $(\mathrm{OR}=6.18,95 \% \mathrm{CI}: 1.71-22.30)$ were significant predictors of higher diagnostic yield. In multivariable analyses, only the presence of a class 2 bronchus sign was a significant predictor for higher diagnostic yield $(\mathrm{OR}=4.83$, 95\% CI: 1.16-20.12). Table S1 summarizes the diagnostic performance of ENB, stratified by the lesion and procedure characteristics. Values of $85.9 \%(61 / 71)$ and $77.3 \%$ $(17 / 22)$ were obtained as the diagnostic yield for lesions in the peripheral two-thirds and one-thirds of the lung, respectively. The diagnostic yield stratified by the bronchus sign was $58.8 \%(10 / 17), 75.0 \%(12 / 16)$, and $89.8 \%(53 / 59)$ for class 0,1 , and 2 bronchus signs, respectively. When analyzing the presence of a class 2 bronchus sign compared to those without (class 0 or class 1 bronchus sign), after adjusting for age, sex, smoking status, and lesion size, the presence of a class 2 bronchus sign was significantly associated with higher diagnostic yield (adjusted $\mathrm{OR}=3.37$, 95\% CI: 1.04-10.95).

\section{Discussion}

In this hospital-based cohort study from South Korea, we determined that sole ENB-guided biopsy under moderate sedation could achieve an overall diagnostic yield of $81.5 \%$ and a sensitivity for malignancy of $77.6 \%$, respectively. ENB showed a good diagnostic performance for peripheral lung lesions. The complication rate was low as $2.1 \%$ for CTCAE grade 2 or greater pneumothorax. Moderate sedation was tolerable for performing $\mathrm{ENB}$, and there were no procedure-related respiratory failure or mortality events. Multivariable analyses revealed the presence of a class 2 bronchus sign as the only significant factor associated with a higher diagnostic yield (OR $=4.83,95 \%$ CI: 1.16-20.12). The diagnostic yield of ENB among lesions with a class 2 bronchus sign was approximately $90 \%$. Our study provides valuable information on the utility and safety of ENB in a least-invasive setting from an Asian population, with use of the Spin Thoracic Navigation System (SYS-4230 K; Veran Medical, St. Louis, MO, USA), of which limited data is available to date. 


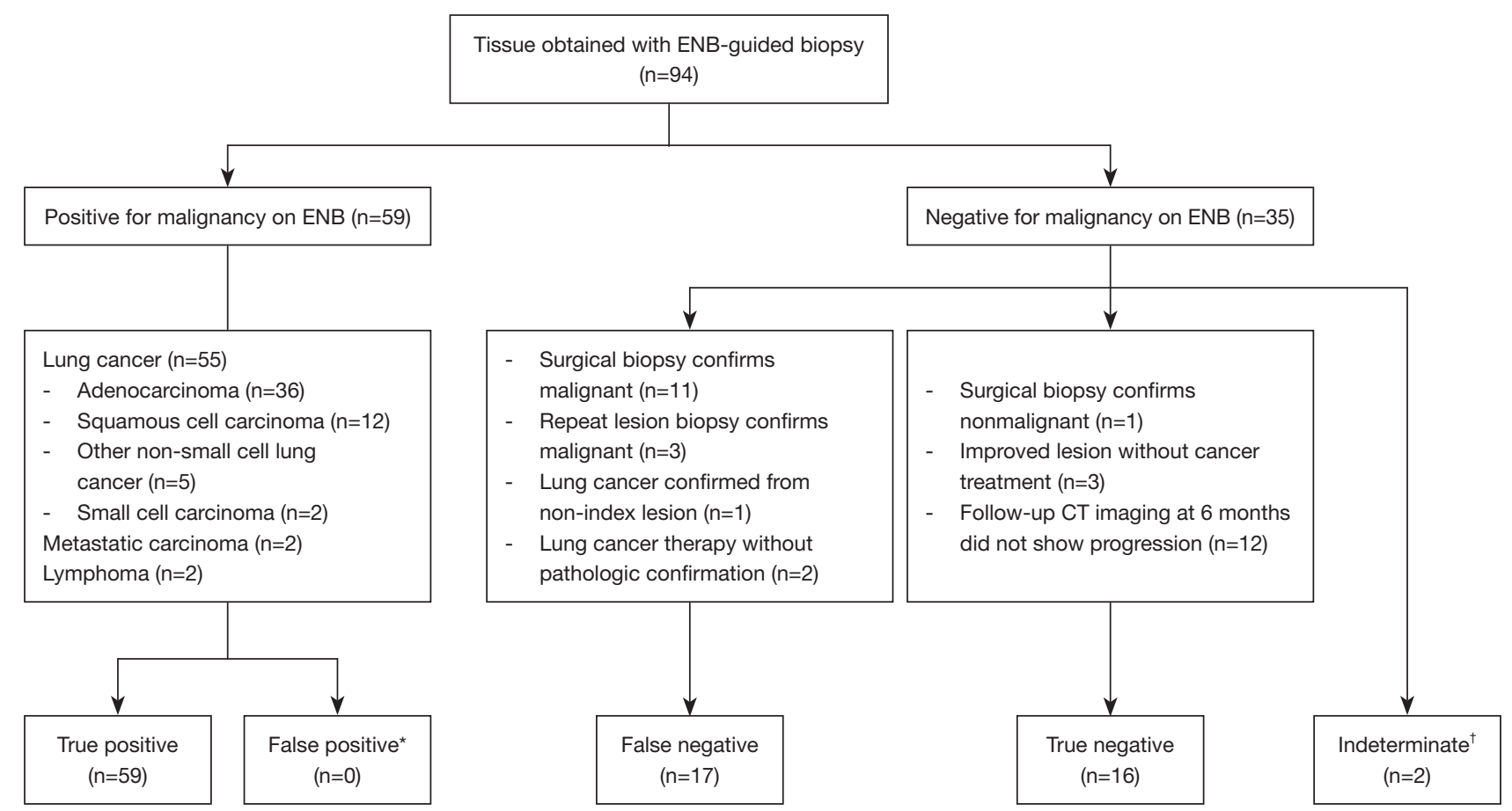

Figure 2 Diagnostic results of participants who underwent electromagnetic navigation bronchoscopy-guided biopsy. *, all malignancy cases confirmed by ENB-guided biopsy were determined as true positives; ${ }^{\dagger}$, initially negative by ENB-guided biopsy and did not complete 6 months follow-up CT imaging for a non-progressive lesion. ENB, electromagnetic navigation bronchoscopy; CT, computed tomography.

Table 2 Diagnostic yield of electromagnetic navigation bronchoscopy

\begin{tabular}{lccc}
\hline & $\begin{array}{c}\text { Excluding indeterminate } \\
\text { cases }(\mathrm{n}=92)\end{array}$ & Low estimate $(\mathrm{n}=94)$ & High estimate $(\mathrm{n}=94)$ \\
\hline Diagnostic yield [(TP + TN)/all biopsies performed] & $81.5 \%(75 / 92)$ & $79.8 \%(75 / 94)$ & $81.9 \%(77 / 94)$ \\
Sensitivity for malignancy & $77.6 \%(59 / 76)$ & $75.6 \%(59 / 78)$ & $77.6 \%(59 / 76)$ \\
Specificity for malignancy & $100 \%(16 / 16)$ & $100 \%(16 / 16)$ & $100 \%(18 / 18)$ \\
Positive predictive value & $100 \%(59 / 59)$ & $100 \%(59 / 59)$ & $100 \%(59 / 59)$ \\
Negative predictive value & $48.5 \%(16 / 33)$ & $45.7 \%(16 / 35)$ & $51.4 \%(18 / 35)$ \\
\hline
\end{tabular}

TP, true positive; TN, true negative.

Current guidelines on the diagnosis of pulmonary lesions recommend that the type of biopsy should be based on the location, size, invasiveness of the available procedure, and the risk of complications $(4,20)$. Recent advances in bronchoscopy techniques, including ENB and r-EBUS, have made transbronchial biopsy more appealing $(7,14)$. Since the first human study performed in 2005, the introduction of ENB has allowed physicians to accurately localize lung lesions and perform biopsies at sites beyond the reach of conventional bronchoscopy through a minimally invasive procedure (21). Various studies on the utility and safety of ENB have reported a diagnostic yield ranging from 33\% to $97 \%$, with most studies reporting the diagnostic yield between $67 \%$ and $84 \%(9,22,23)$. The NAVIGATE study is the largest multi-center study on ENB that reported an overall diagnostic yield of $72.9 \%(10)$. The latest metaanalysis on the performance of ENB evaluating more than 3,000 procedures revealed a pooled sensitivity of $77 \%$ for malignancy (11). However, previous studies have demonstrated marked variability in the setting of the ENB 
Table 3 Univariable and multivariable regression analyses of factors associated with the diagnostic yield of electromagnetic navigation bronchoscopy

\begin{tabular}{|c|c|c|c|c|}
\hline Variable & \multicolumn{2}{|c|}{ Univariate } & \multicolumn{2}{|c|}{ Multivariate } \\
\hline Age & $1.03(0.98-1.09)$ & 0.223 & $1.05(0.99-1.12)$ & 0.120 \\
\hline Female sex (vs. male) & $0.81(0.27-2.46)$ & 0.711 & $0.45(0.04-5.24)$ & 0.526 \\
\hline Smoking history (vs. never smoker) & $0.94(0.32-2.74)$ & 0.911 & $0.35(0.03-3.75)$ & 0.387 \\
\hline Solid type (vs. subsolid) & $2.37(0.40-14.12)$ & 0.345 & & \\
\hline Lower lobe distribution (vs. upper or middle lobe) & $1.69(0.54-5.29)$ & 0.366 & & \\
\hline Distance from visceral pleura in $\mathrm{mm}$ & $1.01(0.97-1.05)$ & 0.616 & & \\
\hline \multicolumn{5}{|l|}{ Bronchus sign (vs. class 0 ) } \\
\hline Use of forceps as biopsy device (vs. needle) & $0.70(0.20-2.36)$ & 0.561 & & \\
\hline Sequential EBUS-TBNA for mediastinal staging & $1.69(0.57-5.05)$ & 0.345 & & \\
\hline Total procedure time in minutes & $0.99(0.96-1.02)$ & 0.552 & & \\
\hline
\end{tabular}

OR, odds ratio; $\mathrm{Cl}$, confidence interval; EBUS, endobronchial ultrasound; TBNA, transbronchial needle aspiration.

procedure $(11,23)$. Although similar diagnostic yield from different navigation systems are reported, most of the studies to date have reported on data from ENB performed with the SuperDimension navigate system. In addition, there are limited data on ENB using the Veran navigate system, particularly those on Asian population with the sole use of ENB under moderate sedation $(9,11)$. In the NAVIGATE study, of the 1,215 participants who underwent ENB, $81.4 \%$ received the procedure under general anesthesia. Complementary techniques included fluoroscopy in $91.0 \%$, r-EBUS in $57.4 \%$, and ROSE in $68.5 \%$ cases, respectively (10). A recent meta-analysis did not reveal differences in pooled sensitivity among groups stratified by the type of sedation and the concurrent use of fluoroscopy, r-EBUS, and ROSE (11). However, it is clear that a majority of physicians who perform ENB in a real-world setting use general anesthesia with various concurrent complementary techniques. Regarding data that show general anesthesia is associated with a greater need for post-procedure escalation of care in bronchoscopy procedures (15), sole ENB under moderate sedation would be the least-invasive technique for this procedure. Moreover, the promising results of high diagnostic yield of sole ENB under moderate sedation reported in our study would be extremely relevant since they support the wide utility of ENB across numerous centers, even those with no access to general anesthesia or other complementary techniques.

Previous ENB studies have identified various factors that contribute to improved diagnostic yield. Factors, such as the lesion size, lesion location, the presence of a bronchus sign, the type of anesthesia, the use of concurrent EBUS or ROSE, the number of biopsy tools used, and user experience have been suggested to be associated with better diagnostic yield; however, statistically significant factors considerably vary among studies $(10,24-28)$. In our study, the relatively larger size of the included lesions could have possibly contributed to good diagnostic performance of ENB; however, multivariable analyses did not confirm lesion size as a significant factor associated with better diagnostic yield. In multivariable analyses, the presence of a class 2 bronchus sign was the only significant factor associated with an improved diagnostic yield. High diagnostic probability by ENB of lesions with a bronchus sign was in accordance with the results of the NAVIGATE study (10). In addition, we further stratified and evaluated bronchus signs with a more practical classification $(16,17)$. In our study, the diagnostic yield of ENB in patients with a class 2 bronchus sign (which refers to type I or II by classification suggested by Tsuboi 
and colleagues) was up to $89.8 \%(29,30)$. Considering the reported diagnostic yield of TTNA ranges from $75 \%$ to $97 \%$ with a meta-analysis rate of $92 \%$ (5), the diagnostic yield of sole ENB under moderate sedation for lung lesions with class 2 bronchus sign shown in our study is comparable to that of TTNA. Moreover, the complication rates of overall pneumothorax of $4.3 \%$ and pneumothorax requiring additional intervention of $2.1 \%$ seen in our study are in accordance with the results of prior studies $(10,11)$, and markedly lower compared with reports of TTNA, which is associated with a pooled pneumothorax rate of $19 \%$ to $25 \%$ $(5,6)$. When performing TTNA, the possible risk of pleural recurrence should be also considered (31). Therefore, our results indicate that sole ENB could be the most effective and least-invasive modality for obtaining tissue biopsy from lung lesions with positive class 2 bronchus sign. Future well-designed prospective randomized controlled trials are required to corroborate our findings and apply them into future guidelines.

There are several limitations to this study. First, this was a single-arm, retrospective study from a single center. Therefore, the results may not be generalizable to other clinical settings. Moreover, our results neither indicate nor validate the utility of ENB over other diagnostic techniques, the physician's decision on patient selection, the type of sedation, or the need to conduct concurrent complementary techniques. However, this study provides valuable information on the use of sole ENB under moderate sedation, which is the environment that a maximal number of physicians would be available to perform the procedure. Despite not providing an answer to what the optimal ENB technique is, this study provides the possibility of ENB being the modality of choice for diagnosing specific pulmonary lesions; and also highlights the need for future large-scale trials. Second, our study could only provide diagnostic data of ENB using single biopsy devices, such as forceps or needles. A combination of two or more devices for biopsy may possibly improve the diagnostic yield; however, there are inconsistent data, and concerns on financial burden increase with the use of various biopsy tools in ENB, thus necessitating a future prospective study.

In conclusion, sole ENB under moderate sedation for diagnosing pulmonary lesions demonstrated good diagnostic yield and safety profile. Our data supports the utility and safety of ENB in a least-invasive setting. Particularly, the impressive performance of ENB in lung lesions with class 2 bronchus sign suggests that sole ENB under moderate sedation could be superior to TTNA for specific types of lung lesions when accounting for similar yields and lower complication rates.

\section{Acknowledgments}

Funding: This study was supported by the Seoul National University Bundang Hospital (grant number: 06-2021-0346).

\section{Footnote}

Reporting Checklist: The authors have completed the STROBE reporting checklist. Available at https://tlcr. amegroups.com/article/view/10.21037/tlcr-21-846/rc

Data Sharing Statement: Available at https://tlcr.amegroups. com/article/view/10.21037/tlcr-21-846/dss

Conflicts of Interest: All authors have completed the ICMJE uniform disclosure form (available at https://tlcr.amegroups. com/article/view/10.21037/tlcr-21-846/coif). The authors have no conflicts of interest to declare.

Ethical Statement: The authors are accountable for all aspects of the work in ensuring that questions related to the accuracy or integrity of any part of the work are appropriately investigated and resolved. This study was conducted in accordance with the declaration of Helsinki (as revised in 2013), and was approved by the Institutional Review Board of Seoul National University Bundang Hospital (IRB No. B-2106-693-104). The Institutional Review Board waived the need for written informed consent from participants, considering the retrospective nature of this study.

Open Access Statement: This is an Open Access article distributed in accordance with the Creative Commons Attribution-NonCommercial-NoDerivs 4.0 International License (CC BY-NC-ND 4.0), which permits the noncommercial replication and distribution of the article with the strict proviso that no changes or edits are made and the original work is properly cited (including links to both the formal publication through the relevant DOI and the license). See: https://creativecommons.org/licenses/by-nc-nd/4.0/.

\section{References}

1. Bray F, Ferlay J, Soerjomataram I, et al. Global cancer statistics 2018: GLOBOCAN estimates of incidence and 
mortality worldwide for 36 cancers in 185 countries. CA Cancer J Clin 2018;68:394-424.

2. Mazzone PJ, Silvestri GA, Patel S, et al. Screening for Lung Cancer: CHEST Guideline and Expert Panel Report. Chest 2018;153:954-85.

3. Silvestri GA, Gonzalez AV, Jantz MA, et al. Methods for staging non-small cell lung cancer: Diagnosis and management of lung cancer, 3rd ed: American College of Chest Physicians evidence-based clinical practice guidelines. Chest 2013;143:e211S-50S.

4. National Comprehensive Cancer Network. NCCN Guidelines. Non-Small Cell Lung Cancer. Version 5.2021. Available online: https://www.nccn.org/professionals/ physician_gls/pdf/nscl.pdf. 2021.

5. DiBardino DM, Yarmus LB, Semaan RW. Transthoracic needle biopsy of the lung. J Thorac Dis 2015;7:S304-16.

6. Heerink WJ, de Bock GH, de Jonge GJ, et al. Complication rates of CT-guided transthoracic lung biopsy: meta-analysis. Eur Radiol 2017;27:138-48.

7. Wang Memoli JS, Nietert PJ, Silvestri GA. Metaanalysis of guided bronchoscopy for the evaluation of the pulmonary nodule. Chest 2012;142:385-93.

8. Ost DE, Gould MK. Decision making in patients with pulmonary nodules. Am J Respir Crit Care Med 2012;185:363-72.

9. Mehta AC, Hood KL, Schwarz Y, et al. The Evolutional History of Electromagnetic Navigation Bronchoscopy: State of the Art. Chest 2018;154:935-47.

10. Folch EE, Pritchett MA, Nead MA, et al. Electromagnetic Navigation Bronchoscopy for Peripheral Pulmonary Lesions: One-Year Results of the Prospective, Multicenter NAVIGATE Study. J Thorac Oncol 2019;14:445-58.

11. Folch EE, Labarca G, Ospina-Delgado D, et al. Sensitivity and Safety of Electromagnetic Navigation Bronchoscopy for Lung Cancer Diagnosis: Systematic Review and Metaanalysis. Chest 2020;158:1753-69.

12. Makris D, Scherpereel A, Leroy S, et al. Electromagnetic navigation diagnostic bronchoscopy for small peripheral lung lesions. Eur Respir J 2007;29:1187-92.

13. Steinfort DP, Bonney A, See K, et al. Sequential multimodality bronchoscopic investigation of peripheral pulmonary lesions. Eur Respir J 2016;47:607-14.

14. Ost DE, Ernst A, Lei X, et al. Diagnostic Yield and Complications of Bronchoscopy for Peripheral Lung Lesions. Results of the AQuIRE Registry. Am J Respir Crit Care Med 2016;193:68-77.

15. Eapen GA, Shah AM, Lei X, et al. Complications, consequences, and practice patterns of endobronchial ultrasound-guided transbronchial needle aspiration: Results of the AQuIRE registry. Chest 2013;143:1044-53.

16. Tokoro Y, Yasuo M, Kobayashi T, et al. Computed tomography-guided bronchoscopy in the diagnosis of small peripheral pulmonary lesions: A retrospective study of 240 examinations in a single academic center. Respir Investig 2016;54:347-54.

17. Ali MS, Sethi J, Taneja A, et al. Computed Tomography Bronchus Sign and the Diagnostic Yield of Guided Bronchoscopy for Peripheral Pulmonary Lesions. A Systematic Review and Meta-Analysis. Ann Am Thorac Soc 2018;15:978-87.

18. National Cancer Institute. Cancer Therapy Evaluation Program Common Terminology Criteria for Adverse Events (CTCAE) v5.0. Available online: https://ctep. cancer.gov/protocolDevelopment/electronic_applications/ ctc.htm. 2017.

19. Folch EE, Bowling MR, Gildea TR, et al. Design of a prospective, multicenter, global, cohort study of electromagnetic navigation bronchoscopy. BMC Pulm Med 2016;16:60.

20. Gould MK, Donington J, Lynch WR, et al. Evaluation of individuals with pulmonary nodules: when is it lung cancer? Diagnosis and management of lung cancer, 3 rd ed: American College of Chest Physicians evidence-based clinical practice guidelines. Chest 2013;143:e93S-e120S.

21. Schwarz Y, Greif J, Becker HD, et al. Real-time electromagnetic navigation bronchoscopy to peripheral lung lesions using overlaid CT images: the first human study. Chest 2006;129:988-94.

22. Gildea TR, Mazzone PJ, Karnak D, et al. Electromagnetic navigation diagnostic bronchoscopy: a prospective study. Am J Respir Crit Care Med 2006;174:982-9.

23. Gex G, Pralong JA, Combescure C, et al. Diagnostic yield and safety of electromagnetic navigation bronchoscopy for lung nodules: a systematic review and meta-analysis. Respiration 2014;87:165-76.

24. Bertoletti L, Robert A, Cottier M, et al. Accuracy and feasibility of electromagnetic navigated bronchoscopy under nitrous oxide sedation for pulmonary peripheral opacities: an outpatient study. Respiration 2009;78:293-300.

25. Jensen KW, Hsia DW, Seijo LM, et al. Multicenter experience with electromagnetic navigation bronchoscopy for the diagnosis of pulmonary nodules. J Bronchology Interv Pulmonol 2012;19:195-9.

26. Oh JH, Choi CM, Kim S, et al. Diagnostic yield and safety of biopsy guided by electromagnetic navigation 
bronchoscopy for high-risk pulmonary nodules. Thorac Cancer 2021;12:1503-10.

27. Eberhardt R, Anantham D, Herth F, et al. Electromagnetic navigation diagnostic bronchoscopy in peripheral lung lesions. Chest 2007;131:1800-5.

28. Seijo LM, de Torres JP, Lozano MD, et al. Diagnostic yield of electromagnetic navigation bronchoscopy is highly dependent on the presence of a Bronchus sign on CT imaging: results from a prospective study. Chest 2010;138:1316-21.

Cite this article as: Kim YW, Kim HJ, Song MJ, Kwon BS, Lim SY, Lee YJ, Park JS, Cho YJ, Yoon HI, Lee JH, Lee CT. Utility and safety of sole electromagnetic navigation bronchoscopy under moderate sedation for lung cancer diagnosis. Transl Lung Cancer Res 2022;11(3):462-471. doi: 10.21037/tlcr-21-846
29. Tsuboi E, Ikeda S, Tajima M, et al. Transbronchial biopsy smear for diagnosis of peripheral pulmonary carcinomas. Cancer 1967;20:687-98.

30. Bellinger C, Poon R, Dotson T, et al. Lesion characteristics affecting yield of electromagnetic navigational bronchoscopy. Respir Med 2021;180:106357.

31. Hong H, Hahn S, Matsuguma H, et al. Pleural recurrence after transthoracic needle lung biopsy in stage I lung cancer: a systematic review and individual patient-level meta-analysis. Thorax 2021;76:582-90. 


\section{Supplementary}

Table S1 Diagnostic yield of electromagnetic navigation bronchoscopy classified by the lesion and procedure characteristics

Diagnostic yield $(n=92)$

\section{Lesion size}

$20 \mathrm{~mm}$

$69.2 \%(9 / 13)$

$>20 \mathrm{~mm}$

$83.5 \%(66 / 79)$

Type of lesion

Solid

$82.6 \%(71 / 86)$

Subsolid

$66.7 \%(4 / 6)$

Location of lesion

Right upper lobe

$72.7 \%(24 / 33)$

Right middle lobe

$100 \%(5 / 5)$

Right lower lobe

$86.7 \%(13 / 15)$

Left upper lobe

$83.3 \%(15 / 18)$

Left lower lobe

$85.7 \%(18 / 21)$

Distance from pleura

$78.6 \%(33 / 42)$

$20 \mathrm{~mm}$

$84.0 \%(42 / 50)$

Lesion in peripheral two-thirds of the lung

$85.9 \%(61 / 71)$

Lesion in peripheral one-thirds of the lung

$77.3 \%(17 / 22)$

Bronchus sign on CT

Class 0

$58.8 \%(10 / 17)$

Class 1 (Tsuboi type III)

$75.0 \%(12 / 16)$

Class 2 (Tsuboi type I/II)

$89.8 \%(53 / 59)$

Tool used

Forceps biopsy

$85.2 \%(23 / 27)$

Needle aspiration biopsy

$80.0 \%(52 / 65)$

$\mathrm{CT}$, computed tomography. 